\title{
Efecto metabólico de la metformina en adolescentes obesas con riesgo de diabetes mellitus tipo 2
}

\author{
BERNARDITA PRADO A..$^{1,2,3}$, VERÓNICA GAETE P. ${ }^{2,3,4,5}$, FRANCISCA CORONA H. ${ }^{2,3,5,6}$, \\ ELDRETH PERALTA V. ${ }^{2,3,6}$, PAULA DONOSO A. ${ }^{2,3,6}$, XIMENA RAIMANN T. ${ }^{5,7}$ \\ 1. Médico, Magíster en Nutrición. \\ 2. Centro de Salud del Adolescente SERJOVEN. \\ 3. Departamento de Pediatría y Cirugía Infantil Oriente, Facultad de Medicina, Universidad de Chile. \\ 4. Pediatra Especialista en Adolescencia. \\ 5. Departamento de Pediatría Clínica Las Condes. \\ 6. Pediatra. \\ 7. Pediatra Nutrióloga.
}

\begin{abstract}
Metabolic effect of metformin in obese adolescents at risk of diabetes mellitus type 2

In adults, metformin promotes weight loss and prevents the development of type 2 diabetes mellitus (DM2). However, these effects have not been demonstrated in adolescents at risk for DM2. Objective: To analyze the anthropometric and metabolic impact of metformin in obese adolescents at risk for DM2. Patients and Methods: A double-blind, placebo-controlled study was conducted in 19 obese female adolescents at risk for DM2. A structured lifestyle intervention with nutritional and exercise education and motivational support was assessed over 3 month with an additional follow up period of 3 months. Subjects were randomized to $500 \mathrm{mg} /$ daily of extended release metformin or placebo. Anthropometric (weight, BMI, waist circumference, blood pressure) and metabolic profiles (glycemia, HOMA, lipid profile, AST, ALT) were compared between both groups at the end of both periods. Results: Metformin treated group showed a significant reduction in weight and body mass index (BMI) compared with placebo group. No improvement in the metabolic risk profile was showed in any group. Conclusion: In this study, metformin therapy in combination with a lifestyle intervention helps to reduce weight and BMI in obese adolescent females at risk for DM2, compared to lifestyle and placebo intervention.
\end{abstract}

(Key words: Metformin, adolescents, obesity, insulin sensitivity, type 2 Diabetes Mellitus).

Rev Chil Pediatr 2012; 83 (1): 48-57

Trabajo recibido el 11 de agosto de 2011, devuelto para corregir el 04 de octubre de 2011, segunda versión el 18 de octubre de 2011, aceptado para publicación el 03 de enero de 2012.

Proyecto financiado con fondos del Concurso de Investigación DA-CLC 2009, de la Dirección Académica de Clínica Las Condes.

Correspondencia a:

Dra. Bernardita Prado Alcalde

bernarditaprado@gmail.com 


\section{RESUMEN}

En adultos, se ha demostrado que la metformina favorece la reducción de peso y previene el desarrollo de Diabetes Mellitus tipo 2 (DM2). Sin embargo, aún no se han determinado estos efectos en población adolescente con riesgo de DM2. Objetivo: Analizar el impacto antropométrico y metabólico de la metformina en adolescentes obesas con riesgo de DM2. Pacientes y Método: Estudio randomizado, doble ciego, en que participaron 19 adolescentes obesas con riesgo de DM2 distribuidas aleatoriamente en dos grupos. Ambos fueron sometidos a un período de tratamiento ( 3 meses) con cambios en estilo de vida y farmacoterapia (dosis diaria de metformina $500 \mathrm{mg}$ de liberación prolongada o placebo, respectivamente), continuado por un período de seguimiento (3 meses). Se compararon las variaciones en antropometría (peso, IMC, circunferencia cintura, presión arterial) y perfil metabólico (glicemia, HOMA, perfil lipídico, GOT y GPT) entre ellos al finalizar ambos períodos. Resultados: El grupo tratado con metformina mostró reducción significativa del peso e IMC. La disminución de IMC fue significativamente mayor que la del grupo que recibió placebo. Ningún grupo demostró mejora en el perfil de riesgo metabólico. Conclusión: La terapia con metformina, combinada con intervención en el estilo de vida, reduce el peso e IMC en adolescentes obesas con riesgo de DM2 en comparación con pacientes que reciben intervención en estilo de vida y placebo.

(Palabras clave: Metformina, adolescentes, obesidad, sensibilidad a la insulina, Diabetes Mellitus tipo 2).

Rev Chil Pediatr 2012; 83 (1): 48-57

\section{Introducción}

La obesidad aumenta el riesgo de desarrollar distintas patologías incluyendo la insulinoresistencia (RI) y Diabetes Melitus tipo 2 (DM2), hipertensión arterial, dislipidemia, enfermedades cardiovasculares, infarto cardiovascular, apnea del sueño, hiperuricemia, gota y osteoartritis ${ }^{1}$.

Durante la adolescencia la acumulación de grasa visceral aumenta y la sensibilidad a la insulina disminuye, lo que se acrecienta en el contexto de obesidad ${ }^{2}$. Estudios sobre población adolescente revelan un preocupante aumento en la prevalencia de obesidad ${ }^{3,4}$ y $\mathrm{DM}^{5-7}$. La pubertad está asociada con resistencia a la insulina, afectando la utilización periférica de glucosa y, en un menor grado, de ácidos grasos. Además, en esta etapa de la vida, el sobrepeso y la obesidad promueven un aumento de la resistencia a la insulina. La obesidad, insulinoresistencia y la historia familiar de DM2 son consideradas como la tríada del factor de riesgo de $\mathrm{DM} 2^{8}$. Un estilo de vida saludable puede prevenir o retrasar la aparición de esta patología, pero suele no ser suficiente.

Estudios clínicos en el tratamiento de obesidad adolescente se han concentrado en el tratamiento basado en dieta y ejercicio, sin embargo, el alto costo financiero de los pro- gramas para la pérdida de peso, junto con la alta tasa de fracaso $^{8}$, posicionan a la farmacoterapia como una opción en el tratamiento de la obesidad ${ }^{4}$.

En este contexto aumenta el interés por el uso temprano de aquellos fármacos que mejoren la tolerancia a la glucosa en esta población de alto riesgo ${ }^{8}$. Esto podría interrumpir el ciclo de aumento de peso-insulinoresistencia, que de otra manera conduciría a la DM2 en la adultez ${ }^{8}$.

La metformina es una biguanida que reduce la glucosa de ayuno al disminuir la producción hepática de glucosa, y disminuye el nivel de insulina mediante la sensibilización de los tejidos periféricos ${ }^{9}$. Se ha demostrado que favorece la reducción de peso y de los niveles plasmáticos de glicemia e insulina ${ }^{10-12}$, así como también prevención en el desarrollo de DM2 $2^{13,14}$ en población adulta con factor de riesgo de DM2. Al mejorar la sensibilidad a la insulina, en comparación con placebo, se ha asociado a una mejoría en el peso, IMC y circunferencia de cintura ${ }^{4}$. Por esto se ha propuesto que el tratamiento con metformina complementaría los efectos de la dieta y el ejercicio para reducir el riesgo de DM2 en pacientes adolescentes con riesgo ${ }^{8,10,12}$. Algunos ensayos clínicos en adolescentes obesos hiperinsulinémicos no diabéticos, han demostrado el efecto de la 
metformina a corto plazo en la reducción de peso $^{15}$ e IMC $^{8,16,17}$, así como también su efecto sobre otros factores de riesgo de DM2, tales como el nivel de insulina y glicemia en adolescentes obesos hiperinsulinémicos no diabéticos ${ }^{10,12,18-22}$. Sin embargo, otros estudios han demostrado que no hay impacto significativo en la pérdida de peso ${ }^{23}$, composición corporal, grasa abdominal ${ }^{11}$ ni en mediciones metabólicas de glucosa ${ }^{18,23}$.

Considerando las limitaciones en la información disponible y que su seguridad a largo plazo es desconocida en adolescentes ${ }^{8,15,20}$ aún no se recomienda el uso de metformina para prevenir DM2 en esta población.

El objetivo de este estudio fue analizar el impacto de la metformina en adolescentes obesas con riesgo de desarrollar DM2 evaluado en términos de antropometría y perfil metabólico, observando su efecto en un posterior período de seguimiento. Se propone que la metformina asociada a dieta y ejercicio tendría un impacto positivo sobre parámetros antropométricos $\mathrm{y}$ metabólicos.

\section{Pacientes y Método}

Se llevó a cabo un estudio clínico, prospectivo, randomizado, doble ciego en el Centro de Salud del Adolescente SERJOVEN entre junio de 2009 y julio de 2010. Participaron 19 adolescentes obesas (IMC $>$ p95 según edad y sexo), post menárquicas, entre 13 y 19 años, con al menos un factor de riesgo de DM2. Los factores de riesgo incluyen pariente de primer o segundo grado con historia de DM2 o alteración en el resultado de los siguientes exámenes dentro de los últimos 6 meses: glicemia de ayuno $\geq 100 \mathrm{mg} / \mathrm{dl}$, glicemia postcarga $\geq 140$ $\mathrm{mg} / \mathrm{dl}$ u HOMA $>3,0^{24}$.

Las pacientes recibían habitualmente atención médica en el centro o fueron derivadas desde los colegios municipales de la comuna Lo Barnechea que estaban en conocimiento del estudio.

Se consideraron criterios de exclusión el diagnóstico de Diabetes Mellitus tipo 1 ó 2, enfermedades renales, hepáticas o respiratorias, alcoholismo, trastornos alimentarios, otros trastornos psiquiátricos que pudieran disminuir la adherencia al tratamiento, hipersensibilidad a la metformina, tratamientos farmacológicos con impacto metabólico o nutricional durante los últimos 3 meses y embarazo.

Cada paciente fue evaluada por un pediatra quien se hizo cargo del cumplimiento de los criterios de inclusión, de la evaluación motivacional (escala medida del 1 al 10, donde el valor 1 correspondía a ausencia de motivación, mientras que el valor 10 correpondía a máxima motivación), de la solicitud de exámenes de laboratorio (detallados más adelante) y del consentimiento informado del paciente y de uno de sus padres o adulto responsable. Las adolescentes reclutadas fueron asignadas al azar en dos grupos (A y B) mediante una secuencia de randomización computacional a cargo de un tercero.

Ambos grupos siguieron un período de intervención de tres meses. La intervención del grupo A consistió en una guía nutricional (de acuerdo a pauta de $1500 \mathrm{kcal} / \mathrm{día})$, programa de ejercicios (clases de gimnasia una vez por semana y una guía de ejercicios para ser practicada dos veces por semana) y metformina en dosis diaria de $500 \mathrm{mg}$ de liberación prolongada. La intervención del grupo B fue la misma, pero en lugar de metformina recibió placebo. Un laboratorio externo estaba a cargo de empaquetar y etiquetar los frascos, manteniendo el conocimiento del contenido bajo confidencialidad hasta que finalizó el estudio. Durante el período de intervención las pacientes se controlaron una vez por semana durante el primer mes y cada dos semanas el segundo y tercer mes. En estas visitas, se midieron parámetros clínicos (descritos más adelante), y la adherencia al tratamiento: dieta (escala likert ${ }^{25}$, cuyo puntaje va de 0 a 32 ), ejercicio (escala likert ${ }^{25}$ con puntaje de 0 a 6 ), asistencia a clases de gimnasia (\% considerando el total de clases ofrecidas como el $100 \%$ ) y medicamento (\% considerando el total de días del período intervencional como $100 \%$ ). En el período de seguimiento de tres meses la frecuencia de los controles se mantuvo. Los exámenes de laboratorio se realizaron al ingreso, a los 3 y 6 meses. El test de tolerancia a la glucosa sólo se realizó al inicio para evaluar criterios de inclusión. La metodología de evaluación de 
los parámetros clínicos y de laboratorio se describe a continuación: a) Peso $(\mathrm{kg})$ con pesa de capacidad para $120 \mathrm{~kg}$, y con aumento de $1 \mathrm{~kg}$, con la adolescente en ropa interior y después de orinar; b) Altura (m) con estadiómetro con una precisión de $0,5 \mathrm{~cm}$, con la adolescente descalzas y el plano Frankfurt horizontal; c) Indice de masa corporal (IMC) por la fórmula peso $(\mathrm{kg}) /$ altura $\left.(\mathrm{mt})^{2} ; \mathrm{d}\right)$ Circunferencia de cintura (CC) con una huincha flexible en centímetros $(\mathrm{cm})$, correspondiendo al perímetro menor entre la cresta ilíaca y el borde inferior de la última costilla, luego de exhalar con los brazos relajados en ambos costados; e) Presión sanguínea sistólica (PAS) y Presión sanguínea diastólica (PAD) (mmHg): con esfingomanómetro manual, ubicando la manga a la altura del corazón y su borde inferior a $2 \mathrm{~cm}$ sobre el pliegue del codo, previamente la adolescente debe haber descansado al menos 5 minutos; f) Perfil lipídico (mg/dl) con autoanalizador Gemini Merck; g) Test de tolerancia a la glucosa $(75 \mathrm{~g})$ y glicemia $(\mathrm{mg} / \mathrm{dl})$ con autoanalizador Gemini Merck; h) Insulinemia (mU/ml) medida por quimioluminiscencia; i) Homeostatic Model Assessment (HOMA) por la fórmula insulinemia $(\mathrm{mU} / \mathrm{mL})$ x glicemia $(\mathrm{mg} / \mathrm{dl}) / 405$; j) Transaminasa glutamico-pirúvica (GPT) y transaminasa glutámico-oxalacética (GOT) (uU/ml) con autoanalizador, Gemini Merck; k) Hemograma Completo con Coulter Counter. GOT y GPT fueron solicitados para descartar eventual efecto secundario a metformina.

Tanto las pacientes como sus padres o adulto responsable otorgaron consentimiento informado para la participación en el estudio. Los procedimientos de esta investigación fueron aprobados por el Comité de Ética Institucional del Hospital Luis Calvo Mackenna.

La muestra calculada mínima requerida fue de 8 pacientes por cada grupo, considerando una desviación estándar de 0,4 en pacientes recibiendo tratamiento ${ }^{21}$, con una diferencia esperada $=0,6$, poder $=90 \%$ y $\mathrm{p}<0,05$. En el análisis de datos se utilizó un intervalo de confianza de $95 \%$ y un valor $\mathrm{p}<0,05$. Se utilizan test estadísticos no paramétricos. Para definir si ambos grupos eran comparables se utilizó test de Wilcoxon signed rank para variables cuantitativas continuas y test de Fisher para variables cualitativas dicotómicas. Para comparar el efecto del tratamiento en cada grupo se utilizó test Wilcoxon signed rank, y para evaluar la diferencia entre los tratamientos se utilizó el test Mann Whitney Rank Sum. Se usó el test Cohen's $d$ para calcular el efecto del tamaño de la muestra. Los resultados se representan como media \pm DS.

\section{Resultados}

Se evaluaron 41 adolescentes inicialmente, de las cuales 26 cumplían los criterios de inclusión al protocolo. De las 26 ingresadas, 19 completaron el período de 3 meses de intervención (10 en el grupo con metformina y 9 en el grupo con placebo) y 7 fueron excluídas durante este período. La exlusión se fundamentó en la inasistencia consecutiva a tres o más controles, inasistencia no consecutiva a cuatro o más controles, o la necesidad de iniciar o descontinuar otro fármaco con potencial impacto nutricional o metabólico. De las 19 pacientes que finalizaron el período intervencional, 13 completaron el siguiente período de seguimiento ( 7 en el grupo con metformina y 6 en grupo con placebo) ya que 6 debieron ser excluídas. La exlusión durante este segundo período se fundamenta en las mismas razones por las que se excluyeron en el primer período.

\section{Características basales}

El promedio de edad de la muestra fue 15,6 $\pm 1,9$ años y la edad de menarquia fue 12,5 $\pm 1,6$ años. Treinta por ciento del grupo con metformina y $44,4 \%$ del grupo con placebo recibían fármacos concomitantemente, como fluoxetina, sertralina, metilfenidato o anticonceptivos hormonales, $30 \%$ del grupo con metformina y $11,1 \%$ del grupo con placebo presentaba comorbilidad psiquiátrica (Trastorno de déficit atencional con hiperactividad y Trastorno Ansioso).

La proporción de pacientes que presentaba los factores de riesgo estudiados se describe en la tabla 1. Ambos grupos eran comparables en relación a las variables analizadas. Sólo una adolescente presentó dos factores de riesgo de DM2.

El 36,8\% de las adolescentes solicitó ingre- 
Tabla 1. Prevalencia de factores de riesgo de Diabetes Mellitus tipo 2 (DM2)

\begin{tabular}{|lcccc|}
\hline Factor de riesgo de DM2 & $\begin{array}{c}\text { Prevalencia } \\
\text { Total } \\
(\mathbf{n = 1 9 )}\end{array}$ & $\begin{array}{c}\text { Prevalencia } \\
\text { Grupo } \\
\text { metformina } \\
\mathbf{( n = 1 0 )} \\
\mathbf{\%}\end{array}$ & $\begin{array}{c}\text { Prevalencia } \\
\text { Grupo } \\
\text { placebo } \\
\text { (n= 9) } \\
\text { \% }\end{array}$ & $\mathbf{p}^{*}$ \\
\hline Pariente de primer o segundo grado con historia de DM2 & 84,2 & 80,0 & 88,0 & 0,42 \\
\hline Glicemia $\geq 100 \mathrm{mg} / \mathrm{dl}$ & 10,5 & 10,0 & 11,0 & 0,52 \\
\hline Glicemia postcarga $\geq 140 \mathrm{mg} / \mathrm{dl}$ & 0 & 0 & 0 & 1,00 \\
\hline HOMA $>3,0$ & 10,5 & 10,0 & 11,0 & 0,52 \\
\hline
\end{tabular}

*Wilcoxon signed rank para variables cuantitativas continuas y test de Fisher para variables cualitativas dicotómicas.

Tabla 2. Resultados de la encuesta motivacional

\begin{tabular}{|c|c|c|c|c|}
\hline Ítem & $\begin{array}{c}\text { Total } \\
\text { (n=19) }\end{array}$ & $\begin{array}{c}\text { Grupo } \\
\text { metformina } \\
(n=10)\end{array}$ & $\begin{array}{c}\text { Grupo } \\
\text { placebo } \\
(n=9)\end{array}$ & $\mathbf{p}^{*}$ \\
\hline Cuán importante es recibir tratamiento & $8,5 \pm 2,0$ & $9,9 \pm 0,3$ & $7,8 \pm 3,2$ & 0,19 \\
\hline Cuánto desea recibir tratamiento & $9,8 \pm 0,4$ & $9,9 \pm 0,3$ & $9,8 \pm 3,1$ & 0,52 \\
\hline Cuán necesario es recibir el tratamiento & $9,4 \pm 1,4$ & $9,5 \pm 1,6$ & $9,2 \pm 3,1$ & 0,33 \\
\hline
\end{tabular}

*Wilcoxon signed rank.

so al protocolo en forma espontánea, $47,3 \%$ fue derivado por su colegio para participar y $10,5 \%$ por sus padres o apoderados. No hubo diferencias significativas entre ambos grupos en la evaluación motivacional. Los resultados de la encuesta motivacional se detallan en la tabla 2.

Una importante proporción de la muestra presentó parámetros de riesgo antropométrico en el período basal. Todas las participantes tenían un $\mathrm{IMC}>\mathrm{p} 95.31,6 \%$ presentó $\mathrm{PAS}>\mathrm{p} 90$ ( $20 \%$ y $44,4 \%$ en grupo con metformina y placebo, respectivamente), 5,3\% presentó PAD $>$ p90 (10\% y $0 \%$, respectivamente) y $78,9 \%$ presentó CC > p90 (90\% y 66,7\%, respectivamente). Así mismo, una proporción significativa de la muestra presentó parámetros metabólicos de riesgo en el período basal: La media de HOMA fue 2, $6 \pm 1,0$ y 2,9 $\pm 1,6$ en el grupo con metformina y placebo respectivamente, $26,3 \%$ presentó HOMA > p75 (30\% y 33,3\%, respectivamente), $5,3 \%$ presentó colesterol $\mathrm{HDL}<40 \mathrm{mg} / \mathrm{dl}(0 \%$ y $11,1 \%$, respectivamente) y $15,8 \%$ presentó triglicéridos $>110 \mathrm{mg} / \mathrm{dl}$ (18\% y $22,2 \%$, respectivamente).

\section{Período de Intervención}

\section{Efecto en perfil antropométrico}

Se compararon las variables antropométricas antes y después del período de intervención en cada grupo. En el grupo con metformina, el efecto del tratamiento fue estadísticamente significativo sobre el peso $(\mathrm{p}=0,01$, $\mathrm{W}-48, \mathrm{~T}+3,5, \mathrm{~T}-\mathrm{51}, 5$, Cohens'd $=0,77$ ) e IMC $(\mathrm{p}=0,002, \mathrm{~W}-55, \mathrm{~T}+0,0, \mathrm{~T}-55,0$, Cohens' $d=0,89)$, no así en el grupo con placebo $(\mathrm{p}=0,25, \mathrm{~W}-21, \mathrm{~T}+12, \mathrm{~T}-33, \mathrm{y} \mathrm{p}=0,164$, $\mathrm{W}-25, \mathrm{~T}+10, \mathrm{~T}-35$ para peso e IMC respectivamente). El resto de las variables estudiadas no evidenció significancia estadística al analizar el efecto de la intervención en cada grupo.

Al comparar la diferencia del efecto del tratamiento entre los grupos, el grupo con metformina evidenció significancia estadística sobre el $\operatorname{IMC}(\mathrm{p}=0,034, \mathrm{U} 19,0, \mathrm{~T} 116$, Cohen's d = 0,5) por sobre el grupo con placebo. No se observó lo mismo con respecto al peso $(\mathrm{p}=0,74, \mathrm{U}$ 40,5, T 94,5). La figura 1 compara el efecto entre ambas intervenciones. No hubo diferencias significativas sobre las otras variables antropo- 


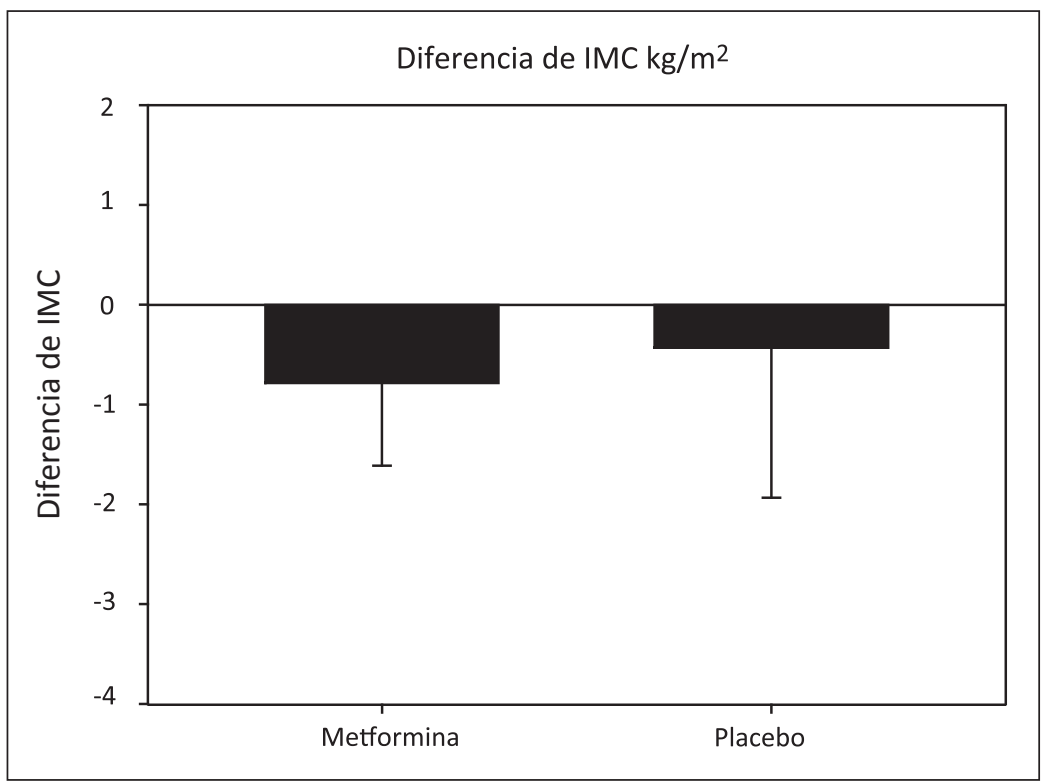

Figura 1. Comparación del efecto de metformina y placebo sobre el IMC al término del período de intervención. métricas al comparar entre los grupos.

\section{Efecto en perfil metabólico}

Al comparar el período basal con el período de intervención se observó un aumento en el nivel de glicemia en ambos grupos, alcanzando significancia estadística sólo en el grupo con metformina (metformina 78,7 $\pm 11,5 \mathrm{y}$ $87,8 \pm 4,5 \mathrm{mg} / \mathrm{dl}$ período basal e intervención, respectivamente, $\mathrm{p}<0,049, \mathrm{~W} 39, \mathrm{~T}+47$, T8,0, Cohen's $d=0,6$; placebo $83,7 \pm 11,5 \mathrm{y}$ $85,6 \pm 4,9 \mathrm{mg} / \mathrm{dl}$, basal e intervención, respectivamente, $\mathrm{p}=0,57, \mathrm{~W} 11, \mathrm{~T}+28, \mathrm{~T}-17$ ). Ambos grupos mostraron una disminución en el colesterol HDL (metformina 51,1 $\pm 10,6$, y 45,2 $\pm 9,2 \mathrm{mg} / \mathrm{dl}, \mathrm{p}<0,055, \mathrm{~W}-32, \mathrm{~T}+6,5$, T- 38,5, Cohen's d =0,6; placebo 55,8 $\pm 15,4$ y 43,1 $\pm 10,8 \mathrm{mg} / \mathrm{dl}, \mathrm{p}<0,004, \mathrm{~W}-45, \mathrm{~T}+0$, T- 45, Cohen's $d=0,9$ ). No se observaron otras diferencias significativas en las variables metabólicas en cada grupo, ni al comparar los resultados entre ambos grupos.

\section{Período de Seguimiento}

\section{Efecto en perfil antropométrico}

A lo largo de los seis meses de estudio, el grupo con metformina mostró una disminución mantenida de peso, IMC y $\mathrm{CC}$, incluso después de finalizado el período de intervención, manteniéndose este efecto a lo largo del período de seguimiento. Por su parte, el grupo con placebo mostró un aumento en estos parámetros después del período de intervención. Sin embargo, ambas tendencias no alcanzaron significancia estadística (figuras 2 y 3 ).

\section{Efecto en perfil metabólico}

Durante el período de seguimiento hubo una tendencia de disminución del nivel de glicemia en el grupo con metformina $(87,8 \pm 4,5$ y $84,0 \pm 40,7 \mathrm{mg} / \mathrm{dl}$, período de intervención y seguimiento respectivamente), sin embargo, no alcanza significancia estadística. Por el contrario, en el grupo placebo se evidenció una tendencia de aumento de esta variable $(85,5$ $\pm 4,9$ y $87,2 \pm 46,2 \mathrm{mg} / \mathrm{dl}$, período de intervención y seguimiento respectivamente). Al comparar ambos grupos, la reducción de glicemia observada en el grupo con metformina fue significativa respecto al aumento del grupo con placebo $(-2,7 \pm 3,2 \mathrm{mg} / \mathrm{dl}$ y $3,8 \pm 4,0 \mathrm{mg} /$ $\mathrm{dl}$, metformina y placebo respectivamente, $\mathrm{p}<$ $0,01, \mathrm{~T} 48)$. No se observaron otras diferencias significativas en otras variables metabólicas en cada grupo. Ambos grupos mostraron un colesterol HDL estable luego de la reducción observada en el período de intervención $(-3,6$ 
PRADO B. y cols.
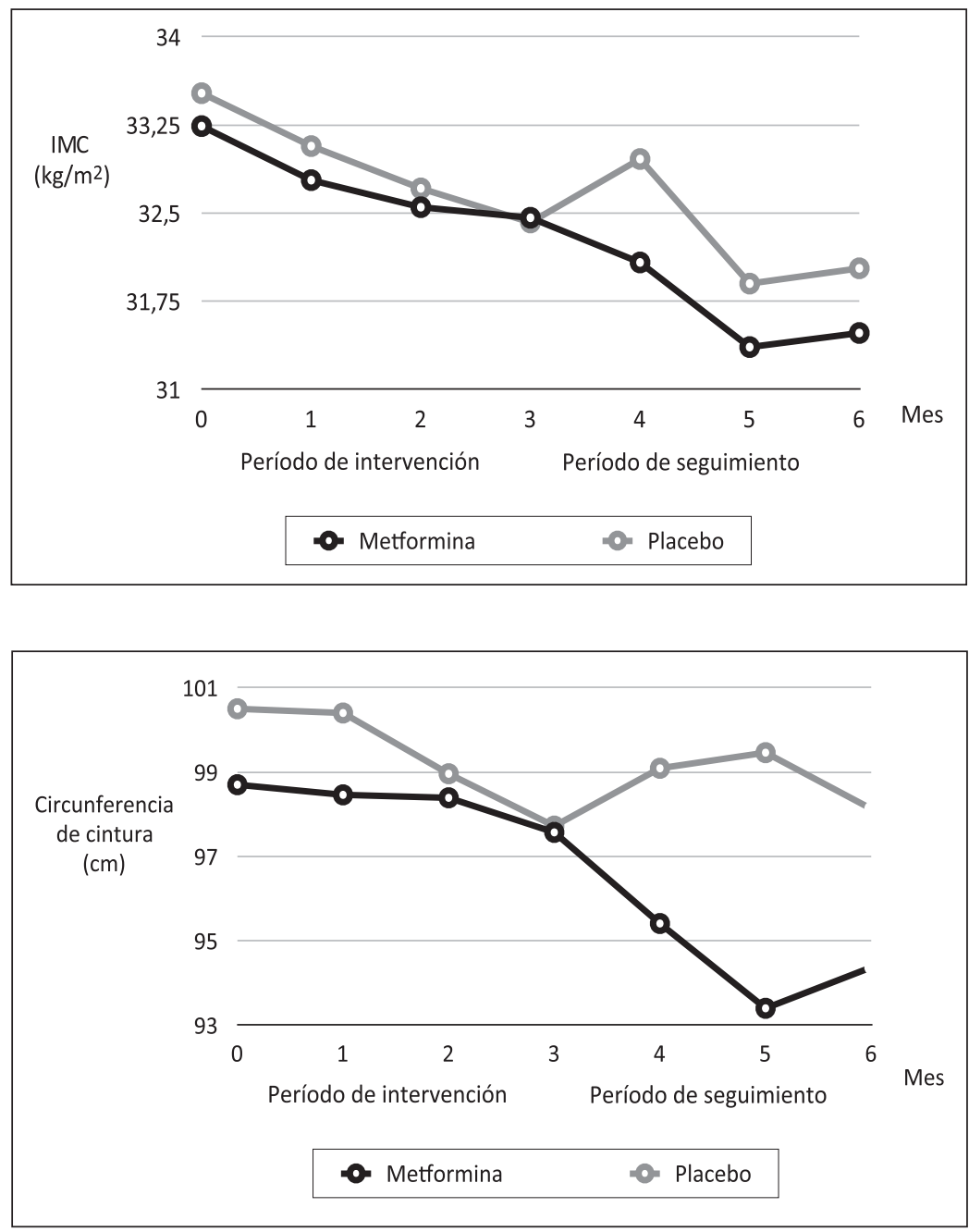

Figura 2. Curva de IMC a lo largo del estudio.
Figura 3. Curva de la circunferencia de cintura a lo largo del estudio.

Tabla 3. Cambios en los niveles de GOT, GPT y hemoglobina con el tratamiento

\begin{tabular}{|lcccccc|}
\hline Variable & \multicolumn{3}{c}{$\begin{array}{c}\text { Grupo metformina } \\
\text { (n= 10) }\end{array}$} & & & \multicolumn{2}{c|}{$\begin{array}{c}\text { Grupo placebo } \\
\text { (n = 9) }\end{array}$} \\
& Basal & Post intervención & $\mathbf{p}^{*}$ & Basal & Post intervención & p * $^{*}$ \\
\hline GOTmU/ml & $12,8 \pm 4,6$ & $18,9 \pm 3,7$ & $<0,008$ & $14,3 \pm 3,3$ & $23,4 \pm 8,6$ & $<0,004$ \\
\hline GPT mU/ml & $11,1 \pm 4,7$ & $19,0 \pm 4,7$ & $<0,002$ & $13,1 \pm 4,4$ & $21,7 \pm 12,3$ & $<0,02$ \\
\hline Hemoglobina mg/dl & $13,7 \pm 0,7$ & $13,1 \pm 0,8$ & $<0,004$ & $14,4 \pm 0,9$ & $13,7 \pm 0,7$ & $<0,016$ \\
\hline
\end{tabular}

*Wilcoxon signed rank.

$\pm 6,6$ y $1,8 \pm 3,5 \mathrm{mg} / \mathrm{dl}$, metformina y placebo respectivamente).

Adherencia a la terapia y efectos secundarios

Durante el período de intervención, la adhe- rencia del grupo con metformina y el placebo fue la siguiente: dieta $24,4 \pm 0,2$ y $23,5 \pm 0,1$ (escala likert ajustada), programa de ejercicio $3,7 \pm 0,5$ y $3,4 \pm 1,0$ (escala likert ajustada), asistencia a clases de gimnasia 23 y $48 \%$, ad- 
herencia diaria al fármaco 92,7 y $88,4 \%$, y la inasistencia a control $2,2 \pm 1,2$ y $2,2 \pm 1,4$ respectivamente. Tanto la metformina como el placebo fueron bien tolerados por las pacientes a lo largo del estudio.

Con respecto a efectos secundarios, después del tratamiento ambos grupos mostraron un aumento significativo en GOT y GPT, y una reducción significativa del nivel de hemoglobina, pero dentro de rangos normales (Tabla $3)$.

\section{Discusión}

Este estudio representa un intento de explorar nuevas estrategias para reducir el riesgo de DM2 en la población adolescente altamente susceptible. Por tanto, fue conducido en adolescentes obesas con parientes en primer o segundo grado con historia familiar de DM2, intolerancia a la glucosa o evidencia de insulinoresistencia.

Se evidenció que 3 meses de terapia con metformina, sumado a una intervención en el estilo de vida, tuvo un efecto favorable en la antropometría, considerando peso $(\mathrm{p}=0,01)$ e $\operatorname{IMC}(\mathrm{p}=0,002)$ con un alto efecto de tamaño muestral. Del mismo modo, el efecto del tratamiento en el IMC también fue estadísticamente significativo al compararlo con el placebo $(\mathrm{p}=0,03)$.

Sin embargo, al igual que en otros estu$\operatorname{dios}^{17,18,23}$, no se encontraron mejoras significativas en la CC, sensibilidad a la insulina y otros parámetros metabólicos. La ausencia de una mejora en la sensibilidad a la insulina ha sido común en algunos estudios con adolescentes, lo que explicaría que este fenómeno puede ser una consecuencia de los cambios de sensibilidad a la insulina que ocurren durante la pubertad ${ }^{18,20}$. En este estudio, la no mejora de la sensibilidad a la insulina después de 3 meses de tratamiento con metformina podría reflejar una pérdida insuficiente de grasa visceral. Esto, considerando que la reducción de la $\mathrm{CC}$ no fue significativa al comparar los valores basales con el período de intervención.

Durante el período de intervención, el gru- po con metformina mostró una reducción en el peso e IMC, efecto que se sostuvo durante el período de seguimiento. En este período se agregó la reducción de la CC. Por el contrario, el grupo placebo mostró un aumento en el peso, IMC y CC después de dicho período. Como se mencionó, no se alcanzó significancia estadística al comparar entre ambos grupos los resultados del período de seguimiento. Probablemente esto se explicaría en la reducción del tamaño muestral observado en esta etapa del estudio.

Durante el período de intervención, la glicemia evidenció un aumento en el grupo con metformina. Sin embargo, esto se revirtió a lo largo del período de seguimiento, en el cual se observó una significativa reducción de la glicemia $(\mathrm{p}=0,01)$. Estudios clínicos con adolescentes describen una reducción de la glicemia tras una terapia de 6 meses con metformi$\mathrm{na}^{8,18-22}$. Probablemente cualquier tratamiento de menor duración no sería suficiente para alcanzar este efecto.

Estudios indican que la metformina presenta efectos antiinflamatorios y lipolíticos ${ }^{26,27}$. El presente estudio no apoyó esta propiedad del fármaco. No se observó diferencia significativa en el perfil de lípidos entre el grupo metformina y placebo. De hecho ambos grupos mostraron una reducción significativa del colesterol HDL durante el período de intervención (grupo con metformina $\mathrm{p}=0,055$ y grupo placebo $\mathrm{p}=0,004)$, alcanzando estabilización a lo largo del período de seguimiento. Estos resultados son similares a aquellos estudios clínicos que sugieren que un balance calórico negativo y pérdida de peso generan una disminución en el colesterol HDL ${ }^{28}$.

Un tratamiento de largo plazo con metformina puede asociarse a una disminución en la absorción intestinal de vitamina B12 y ácido fólico ${ }^{29,30}$. Se observó que la hemoglobina disminuyó en ambos grupos (metformina, $p=0,004$ y el placebo, $p=0,016)$, siempre dentro de rangos normales. Una vez finalizado el período de intervención, ambos grupos evidenciaron una tendencia hacia el aumento de esta. La determinación de los niveles de vitamina B12 y ácido fólico podría tener utilidad al momento de analizar la disminución 
de hemoglobina.

Aunque siempre dentro de los rangos de normalidad, ambos grupos experimentaron un aumento significativo de transaminasas ( $p$ $=0,008$ ) que revirtió una vez finalizado el período de intervención. El efecto inicial podría deberse al ejercicio, aumento en el consumo de proteínas y/o el uso de fármaco con metabolismo hepático.

Es importante considerar que los resultados obtenidos corresponden a una muestra homogénea. La razón para esta decisión fue adecuar las indicaciones de la terapia para un grupo que presenta un metabolismo similar. Esto debe cautelarse para evitar la generalización de los hallazgos.

Finalmente, a pesar de los esfuerzos por optimizar el seguimiento, la tasa de deserción fue significativa. Este hecho destaca el gran desafío que involucra la implementación de programas de pérdida de peso para los adolescentes de alto riesgo. Se necesitan estudios más prolongados en el tiempo para definir el efecto de esta terapia sobre la sensibilidad a la insulina y tejido graso visceral en adolescentes. Resulta relevante cómo el efecto de la metformina en el peso, IMC y CC permanece una vez finalizado el período de intervención, e incluso más allá del período de 3 meses de seguimiento. También fue significativa la reducción de la glicemia inducida por la metformina en el período de seguimiento. Se necesitan más estudios para explorar el potencial efecto a largo plazo de la metformina en esta población.

Todos estos hallazgos apoyan el uso de metformina como un agente útil para promover pérdida de peso a corto plazo en adolescentes obesas, en asociación a cambios en el estilo de vida.

En conclusión, este estudio revela que la terapia de tres meses con metformina, en combinación a una intervención en el estilo de vida, es efectiva en la reducción de peso e IMC en adolescentes obesas con riesgo de desarrollar DM2. Esto no se observó en el grupo que recibió placebo en combinación con la intervención en el estilo de vida. La diferencia del IMC fue significativamente mayor en el grupo que recibió metformina respecto al que recibió placebo. Tras un período de seguimiento se observó que el efecto de la metformina sobre el peso e IMC se mantuvo, observándose también un a reducción en la circunferencia de cintura significativa.

\section{Agradecimientos}

Los autores agradecen a la Corporación SERJOVEN por aportar la infraestructura y el equipamiento para realizar el estudio, asi como también por facilitar el acceso a los adolescentes que participaron en él. Además, agradece a Laboratorio Chile, Laboratorio Biofórmula, Laboratorio LEP (Especialidades Pediátricas) y Laboratorio IEM (Especialidades Médicas) por su colaboración.

\section{Referencias}

1.- Khaodhiar L, McCowen KC, Blackburn GL: Obesity and its comorbid conditions. Clin Comerstone 1999; 2: 17-31.

2.- Huang TT, Johnson MS, Figueroa-Colon R, Dwyer JH, Goran MI: Growth of visceral fat, subcutaneous abdominal fat, and total body fat in children. Obes Res 2001; 9: 283-9.

3.- Rodríguez L: Situación nutricional del escolar y adolescente en Chile. Rev Chil Pediatr 2007; 78: 523-33.

4.- Pinhas-Hamiel O, Lerner-Geva L, Cooperman N, Jacobson M: Insulin Resistance and parenteral obesity as predictors to response to therapeutic life style change in obese children and adolescents 10-18 years old. J Adol Health 2008; 43: 437-43.

5.- Sinha R, Fisch G, Teague B, et al: Prevalence of impaired glucose tolerance among children and adolescents with marked obesity. N Engl J Med 2002; 346: 802-10.

6.- Barja S, Arteaga A, Acosta A. Resistencia insulínica y otras expresiones del síndrome metabólico en niños obesos chilenos. Rev Med Chile 2003; 131: 259-68.

7.- Krebs NF, Jacobson MS: Prevention of pediatric overweight and obesity. Pediatrics 2003; 112: 424-30.

8.- Freemark M, Bursey D: The effects of metformin on body mass index and glucose tolerance in obese adolescents with fasting hyperinsulinemia and a family history of type 2 diabetes. Pediatrics 2001; 107: 1-7.

9.- Lillioja S, Mott DM, Spraul M: Insulin resistance and insulin secretory dysfunction as precursors of noninsulin dependent mellitus: prospective studies in Pima 
Indians. N Engl J Med 1993; 329: 1988-92.

10. Glaser Pediatric Research Network Obesity Study Group: Metformin extended release treatment of adolescent obesity: A 48 week randomized, double blind, placebo-controlled trial with 48-week follow-up. Pediatr Adolesc Med 2010; 164: 116-23.

11.- Charles MA, Eschwege E, Grandmottet P, et al: Treatment with metformin of non-diabetic men with hypertension, hypertriglyceridaemia and central fat distribution: the BIGPRO 1.2 trial. Diabetes Metab Res Rev 2000; 16: 2-7.

12.- Yanovski J, Krakoff J, Salaita C, et al: Effects of metformin on body weight and body composition in obese insulin-resistant children. A randomized clinical trial. Diabetes 2011; 60: 477-85.

13.- Diabetes Prevention Program, NIDDK, NIH 2008, Publication 09-5099. http://diabetes.niddk.nih.gov/dm/ pubs/preventionprogram. Última visita 30-03-2011.

14.- Ratner RE: An update on the Diabetes Prevention Program. Endocr Pract 2006; 12: 20-4.

15.- Kay JP, Alemzadeh R, Langley G, D'Angelo L, Smith P, Holshouser $S$ : Beneficial effects of metformin in normoglycemic morbidly obese adolescents. Metabolism 2001; 50: 1457-61.

16.- Park MH, Kinra S, Kirsten J, et al: Metformin for obesity in children and adolescents: a systematic review. Diabetes Care 2009; 3: 1743-5.

17.- Park MH, Kinra S: Metformin treatment for adolescent obesity has limited long-term benefits. Arch Pediatr Adolesc Med 2010; 164: 116.

18.- Clarson CL, Mahmud FH, Barker JE, et al: Metformin in combination with structured lifestyle intervention improved body mass index in obese adolescents, but did not improve insulin resistance. Endocrine 2009; 36: 141-6.

19.- Rodríguez J, Shearer B: Metformin therapy and diabetes prevention in adolescents who are obese. Am Fam Physician 2007; 76: 1357-8.

20.- Srinivasan S, Ambler R, Baur L, et al: Randomized, controlled trial of metformin for obesity and insulin resistance in children and adolescents: improvement in body composition and fasting insulin. J Clin Endocrinol Metab 2006; 91: 2074-80.

21.- Atabek ME, Pirgon O: Use of metformin in obese adolescents with hyperinsulinemia: a 6-month, randomized, double-blind, placebo-controlled clinical trial. J Pediatr Endocrinol Metab 2008; 21: 339-48.

22.- Desilets AR, Dhakal Karki S, Daunican K: Role of metformin for weight management in patients without type 2 diabetes. Ann Pharmacother 2008; 42: 817-26.

23.- Love-Osborne K, Sheeder J, Zeitler P: Addition of metformin to a lifestyle modification program in adolescents with insulin resistance. J Pediatr 2008; 152: 817-22.

24.- Burrows R, Leiva L, Burgueño $M$, et al: Sensibilidad insulínica determinada a través de HOMA y Quicki en niños de 6 a 15 años: asociación con factores biológicos. Rev Med Chile 2006; 134: 1417-26.

25.- Jamieson S: Likert scales: how to (ab) use them. Medial Education 2004; 38: 1212-8.

26.- Ibáñez L, Valls C, Cabré S, de Zegher F: Flutamidemetformin plus ethinylestradiol-drospirenone for lipolysis and antiatherogenesis in young women with ovarian hyperandrogenism: the key role of metformin at the start and after more than one year of therapy. J Clin Endocrinol Metab 2005; 90: 39-43.

27.- Ibáñez L, Fucci A, Valls C, Ong K, Dunger D, de Zegher $F$ : Neutrophil count in small-for-gestational age children: contrasting effects of metformin and growth hormone therapy. J Clin Endocrinol Metab 2005; 90: 3435-9.

28.- Shai I, Schwarzfuchs D, HenKin $Y$, et al: Weight loss with a low-carbohydrate, mediterranean, or low-fat diet. N Engl J Med 2008; 359 (3): 229-41.

29.- Aarsand AK, Carlsen SM: Folate administration reduces circulating homocysteine levels in NIDDM patients on long-term metformin treatment. J Intern Med 1998; 244 : 169-74.

30.- De Jager J, Kooy A, Lehert P, et al: Long term treatment with metformin in patients with type 2 diabetes and risk of vitamin B-12 deficiency: randomized placebo controlled trial. BMJ 2010; 340: 2181. 